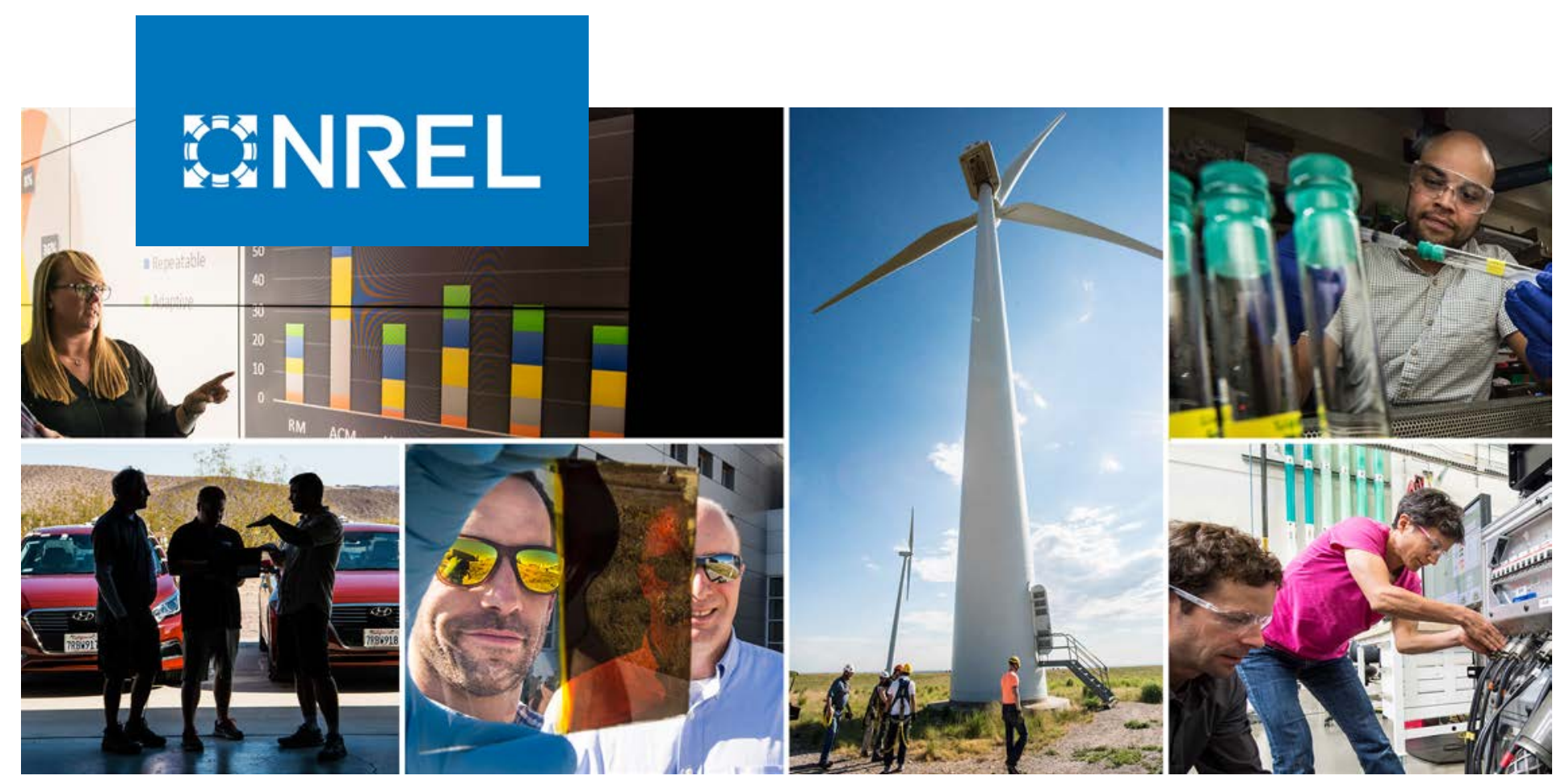

2020 Transportation Research Board

Workshop: Infrastructure Spatial

Sensing at Intersections: The Potential

to Enhance Safety and Enable Automation

Lei Zhu

National Renewable Energy Laboratory

NREL is a national laboratory of the U.S. Department of Energy

Office of Energy Efficiency \& Renewable Energy

Operated by the Alliance for Sustainable Energy, LLC

This report is available at no cost from the National Renewable Energy Laboratory (NREL) at www.nrel.gov/publications.
Technical Report

NREL/TP-5400-76090

April 2020 


\section{GNREL}

2020 Transportation Research Board

Workshop: Infrastructure Spatial

Sensing at Intersections: The Potential

to Enhance Safety and Enable

Automation

\section{Lei Zhu}

National Renewable Energy Laboratory

\section{Suggested Citation}

Zhu, Lei. 2020. 2020 Transportation Research Board Workshop: Infrastructure Spatial Sensing at Intersections: The Potential to Enhance Safety and Enable Automation.

Golden, CO: National Renewable Energy Laboratory. NREL//TP-5400-76090.

https://www.nrel.gov/docs/fy20osti/76090.pdf.

NREL is a national laboratory of the U.S. Department of Energy Office of Energy Efficiency \& Renewable Energy Operated by the Alliance for Sustainable Energy, LLC

This report is available at no cost from the National Renewable Energy Laboratory (NREL) at www.nrel.gov/publications.

Contract No. DE-AC36-08GO28308
Technical Report NREL/TP-5400-76090 April 2020

National Renewable Energy Laboratory 15013 Denver West Parkway Golden, CO 80401

303-275-3000 • www.nrel.gov 


\section{NOTICE}

This work was authored by the National Renewable Energy Laboratory, operated by Alliance for Sustainable Energy, LLC, for the U.S. Department of Energy (DOE) under Contract No. DE-AC36-08GO28308. Funding provided by U.S. Department of Energy Office of Energy Efficiency and Renewable Energy, Vehicle Technologies Office. The views expressed herein do not necessarily represent the views of the DOE or the U.S. Government.

This report is available at no cost from the National Renewable Energy Laboratory (NREL) at www.nrel.gov/publications.

U.S. Department of Energy (DOE) reports produced after 1991 and a growing number of pre-1991 documents are available free via www.OSTI.gov.

Cover Photos by Dennis Schroeder: (clockwise, left to right) NREL 51934, NREL 45897, NREL 42160, NREL 45891, NREL 48097, NREL 46526.

NREL prints on paper that contains recycled content. 


\title{
2020 Transportation Research Board Workshop: Infrastructure Spatial Sensing at Intersections: The Potential to Enhance Safety and Enable Automation
}

\author{
Lei Zhu \\ National Renewable Energy Laboratory (NREL), Lei.Zhu@nrel.gov \\ Coordinated by the National Renewable Energy Laboratory (NREL), Sunday, January 12, 2020
}

The sensing technology that is enabling vehicle automation also can revolutionize traffic intersection safety, control, and efficiency. Infrastructure spatial-sensing technology refers to the deployment of sensors that can detect and track all objects in the field of view-for example, mounting LiDAR (light detection and ranging) sensors, radar, and video imaging at an intersection and fusing the data to produce a threedimensional dynamic operational awareness. Combining such sensing capability with fail-safe vehicle communications can facilitate applications such as eco-approach and departure, optimizing efficient vehicle-signal coordination, and reducing traffic accidents through increased safety. Furthermore, evidence is mounting suggesting that infrastructure-based sensing is needed in order to safely deploy automated public mobility alongside the normal vehicle and pedestrian traffic in our cities. Six speakers were invited to present their work from various aspects (safety, efficiency, and automation) addressing infrastructurebased sensing at the workshop on Infrastructure Spatial Sensing hosted in Washington, D.C. as part of the Transportation Research Board Annual Meeting on January 12, 2020. The details of the workshop, including a brief agenda, can be found at https://annualmeeting.mytrb.org/Workshop/Details/13387. A series of presentations touched on the full envelope of possible benefits from Infrastructure Spatial Sensing. Topics covered included:

- Intersection collision avoidance resulting from dilemma zone monitoring

- Traffic and pedestrian applications from LiDAR deployment at intersections

- Estimating the energy benefits of crash avoidance

- Deconflicting roadway intersections for automated vehicle (AV) "interlocking"

- Advancements in video detection for traffic applications at intersections

- Leveraging sensor-based detection for signal operations in Colorado Springs, CO.

A summary of these presentations is provided below.

\section{Intersection Collision Avoidance Safety Program (iCASP) - Lakeland, Florida} Mr. Angelo Rao, City of Lakeland

The Intersection Collision Avoidance Safety Program (iCASP), which complements the City of Lakeland Police Department's red-light-running safety program, is designed to predict red-light-running motorists using traffic light mounted sensors that can monitor speed with respect to intersection approach, and in relation to signal phase changes. If the sensors detect an impending red-light-running event, the system extends the "all-red" signal phase (perpendicular green light will be delayed) to avoid red-light-running hazard exposure and eventual crashes.

In 2017, nearly half (46\%) of those killed in red-light-running crashes were passengers. More than 5\% were pedestrians or cyclists, and over $35 \%$ of those killed were the drivers who ran the red light. The Heinrich 300-29-1 model ("Safety Triangle") shown in Figure 1 reveals that unsafe behavior patterns lead to 
significant adverse incidents, and the near misses (if monitored) can indicate significantly unsafe conditions and behavior before they result in crashes.

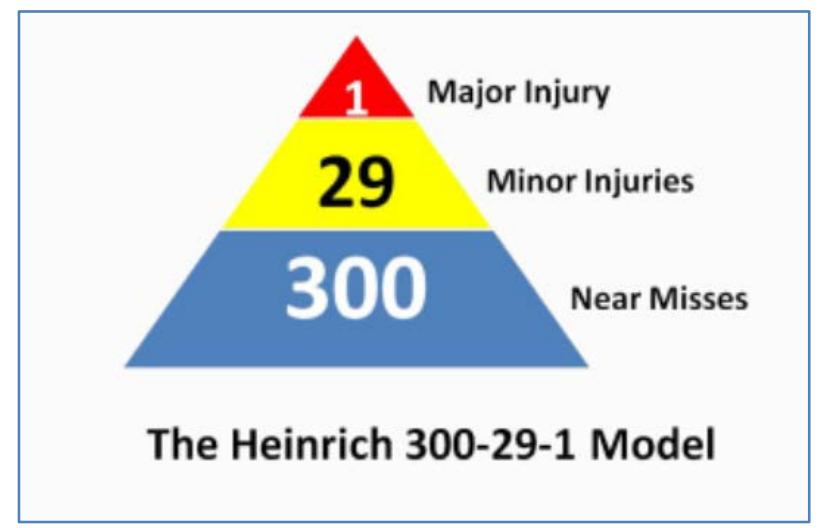

Figure 1. The Heinrich "Safety Triangle"

The deployed system in Lakeland can trigger video recording of hazardous situations, capturing near-miss scenarios, several of which were viewed in the presentation. The instrument suite supporting the demonstration is shown in Figure 2. Statistical analysis for red-light running at intersections in the pilot site is ongoing, but the traffic levels are not significant enough for statistical confidence of system impact based wholly on crash reduction. Lakeland is working with the state department of transportation (DOT) to deploy the system on high-volume (and high crash frequency) intersections in order to statistically demonstrate the effectiveness to sense and avoid dangerous red-light-running hazards.



Figure 2. iCASP system with Iteris Vector sensor and Econolite Cobalt controller

\section{Proof of Concept of Roadside LiDAR Deployment and Applications Dr. Hao Xu, Associate Professor, University of Nevada, Reno (UNR)}

New traffic systems and applications require traffic flow information with more detail and higher accuracy. 360-degree LiDAR sensors deployed on the roadside can be a revolutionary solution because they detect 
surrounding objects with high accuracy and are not influenced by ambient light conditions. Dr. Xu and his colleagues at UNR developed innovative data-processing algorithms and models, particularly for roadside LiDAR sensing systems, and the evaluation of the accuracy, reliability, and efficiency of such systems for various traffic scenarios and applications.

The UNR research team has installed two permanent LiDAR pilot systems, which cover two intersections in Reno, Nevada, and three intersections at Henderson (near Las Vegas), as shown in Figure 3(a). In addition, Dr. Xu has conducted a portable sensor deployment with the Nevada DOT using trailer mounts as well as with tripod mounts, as shown in Figure 3(b).

New applications of LiDAR demonstrated by UNR include an Automatic Rectangular Rapid Flash Beacon (RRFB) control. UNR collected LiDAR and video data from 5 a.m. on September 15, 2019, to 10 p.m. on September 17, 2019, at Green Valley Parkway to evaluate the automatic turning-on performance of RRFB. It implied about a $99.5 \%$ accuracy rate for pedestrian detection.

\section{Deployment Methods - Permanent Installation}

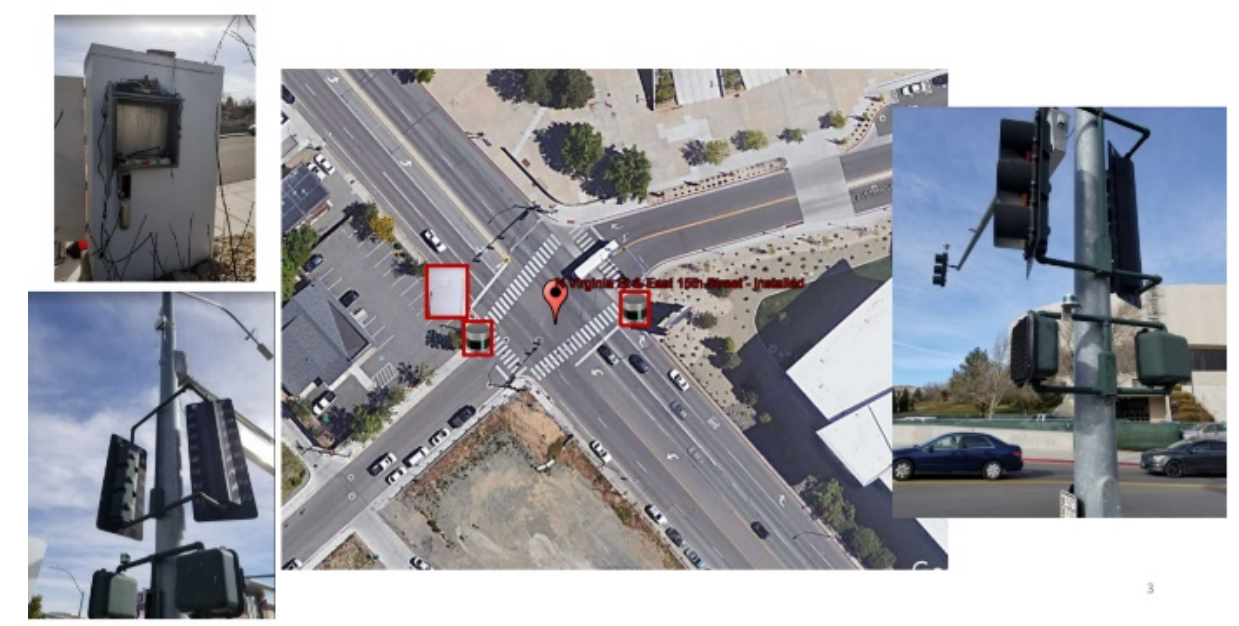

(a)

\section{Deployment Methods - Portable Platform}
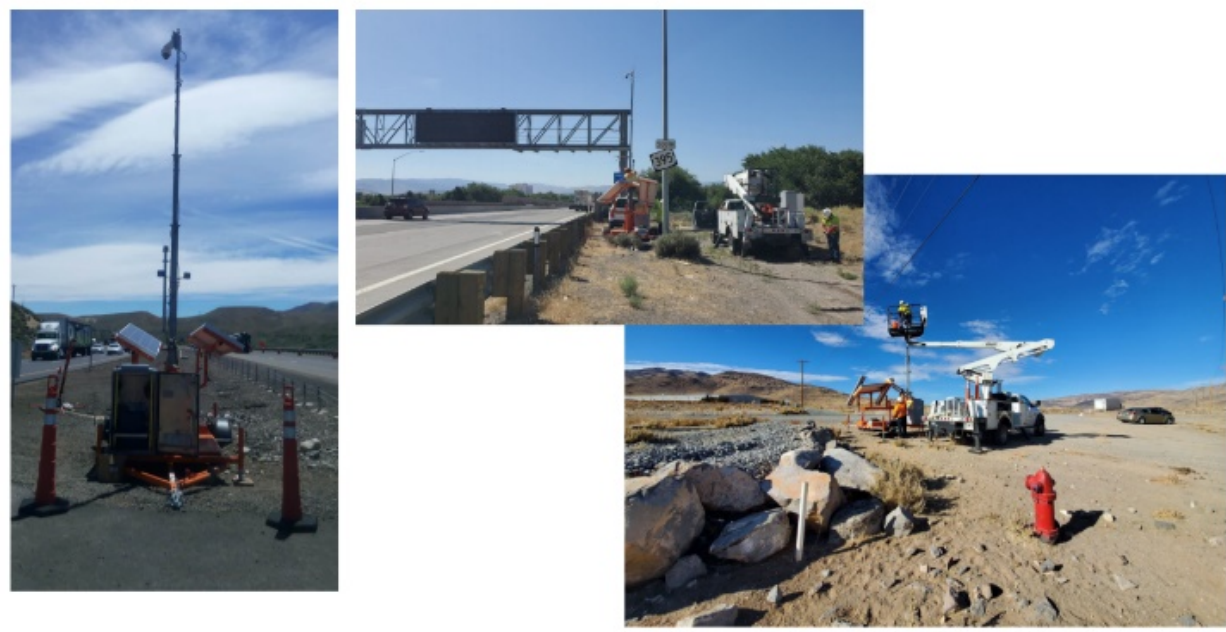

(b)

Figure 3. LiDAR deployment methods 


\title{
A GDP-Weighted First-Order Approximation Framework to Estimate the Energy Equivalence of Safety
}

\author{
Dr. Gary (Zijia) Zhong, National Renewable Energy Laboratory
}

Dr. Zhong presented a framework to estimate the gross domestic product (GDP)-weighted energy equivalence of safety (EES) at intersections based on previous studies of the economic impact of crashes, combined with national-level energy consumption data. Using the ratio of GDP to the total annual energy consumption, highly researched economic impacts were converted to EES impacts for various crash severity levels. The framework, illustrated in Figure 4, explores EES insights through multiple years from 2010 to 2017, and assesses the potential GDP-weighted energy benefits of infrastructure spatial sensing at intersections resulting from increased safety. The energy equivalence of safety study suggests, and underscores, that crash avoidance is paramount at intersections, and systems that can reduce crash frequency will have an order of magnitude (or more) impact on GDP-weighted equivalent energy compared to the direct energy efficiency improvements from reducing stops and smoothing traffic flow.

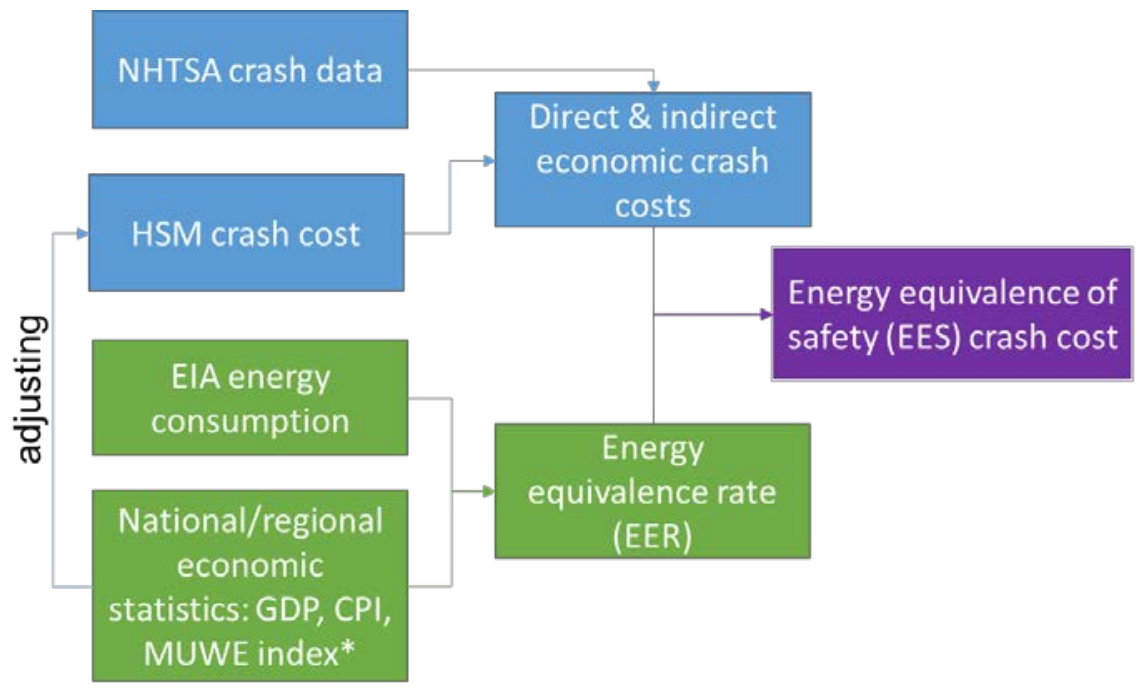

Figure 4. Framework for estimating the GDP-weighted energy equivalence of safety (EES). NHTSA = National Highway Traffic Safety Administration; HSM = Highway Safety Manual; EIA = U.S. Energy Information Administration; CPI = Consumer Price Index; $M U W E=$ median usual weekly earnings

This approach provides a holistic view of the value of safety and energy, which is compatible with hazard exposure and embodied energy of human life and can be applied to national, state, and local levels of crash analysis. The total GDP-weighted EES for the United States is estimated at 17.24 billion gasoline gallon equivalent (GGE) in 2017, while the energy costs per fatal crash are valued at 200,259 GGE in 2017. Figure 5 illustrates the contrast between of fuel wasted by congestion (recurring and nonrecurring congestion) as reported by the Urban Mobility Report as compared to the comprehensive GDP-weighted equivalent energy impacts of all fatal, bodily injury, and property-damage-only crashes in 2017. 


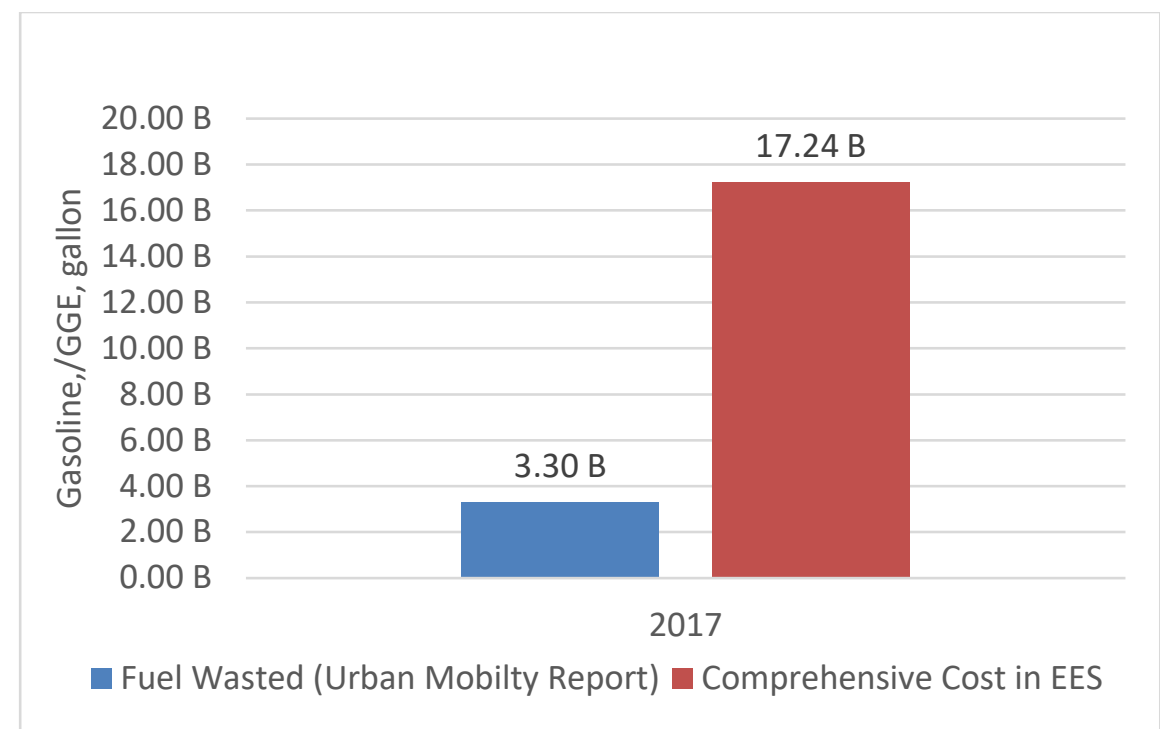

Figure 5. National-level fuel wasted from congestion compared to comprehensive GDP-weighted crash equivalent energy impact in GGE for 2017

\section{Open-Architecture "Interlocking" of Roadway Intersections}

\section{Mr. J. Sam Lott, Principal of Automated Mobility Services, LLC}

Fully automated and unmanned transport systems that utilize communications technology as an integral part of the control system have been in operation for decades, in the form of automated train control and automated people movers (prevalent at large airports). The safety design approach utilizing interlocking zones to protect guideway junctions from unsafe vehicle incursions provides a replicable model for safe application of AV transport technology on roadways, particularly when operated in managed fleets. The concept of "interlocking" as an independent and failsafe subsystem deployed at each roadway intersection is a relevant safety approach for AV fleets operated for public mobility within a geofenced area, defined as an automated mobility district (AMD).

The concept is proposed with an open architecture. The infrastructure-based spatial-sensing technology coupled with mission-critical communications between vehicles and infrastructure creates a roadway junction "interlocking" - also called a "smart intersection." Such a junction interlocking is the basis for the safe deployment of AV for public mobility in mixed traffic. Figure 6 illustrates the safety principles from Communications-Based Train Control (CBTC) as they could be considered for AV fleet applications, as well as the distribution of command and control among the functional subsystems.

A case study of the Houston University District considers a set of characteristic capabilities for safety that parallel those in Figure 6. Anticipating a multi-fleet operating environment within an AMD, consideration has been given to the case of a variety of AV technologies in operation-each having a unique automatic command and control system. Safe operations through a signalized intersection that is equipped with the sensory and artificial intelligence (AI) perception technology as part of the roadway infrastructure (described above as a smart intersection), requires that all technologies and fleets comply with a common vehicle-to-infrastructure (V2I) communications standard and have a common response protocol to the smart intersection's command and control directives. This concept would create the fail-safe technology application for the roadway intersection that is equivalent to guideway switch-interlocking protection defined in CBTC automatic train control standards. 


\section{Functional Safety of Automatic Control Systems/Subsystems}

\section{AV Automatic Fleet Operations Control System}

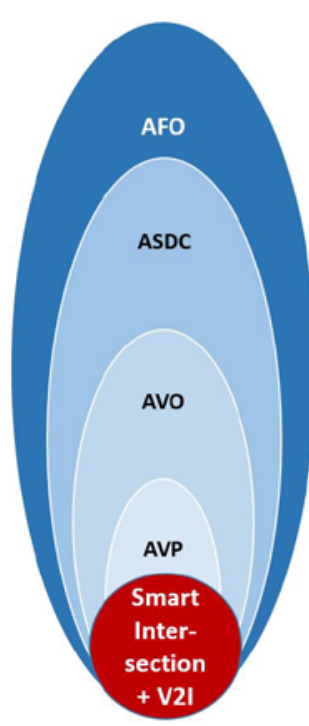

CBTC Automatic Train Control System IEEE 1474 Communications Based Train Control (CBTC)

ATC - Automatic Train Control
$\begin{gathered}\text { System controls train movement, enforces } \\ \text { train safety and directs train operation. }\end{gathered}$
$\begin{gathered}\text { ATS - Automatic Train Supervision } \\ \text { individual trains to maintain schedule. }\end{gathered}$
$\begin{gathered}\text { ATO - Automatic Train Operation } \\ \text { Regulates speed, programmed stopping, } \\ \text { door control, performance level regulation. }\end{gathered}$
ATP - Automatic Train Protection
Maintain fail-safe protection against
collision, excessive speed and other
hazardous conditions (train detection,
train separation and

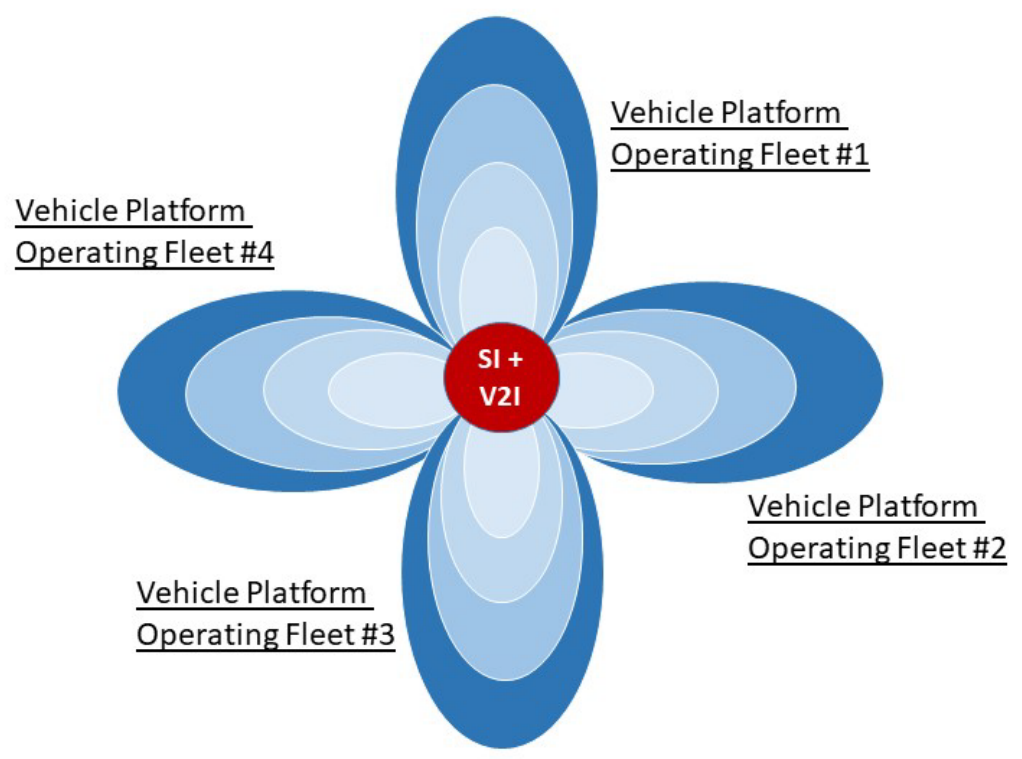

Figure 6. Safety principles from communications-based train control applied to automated vehicle operating environments; (top) Safety of automatic control systems and their functional subsystems; (bottom) Safety of roadway intersection operations for multiple AV technology platforms and diverse operating fleets. Note: SI = smart intersection, ADS = automated driving systems, $D D T=$ dynamic driving task, $V 2 I=$ vehicle to infrastructure 


\title{
Advancements in Using Video for Multimodal Detection and Spatial Understanding at Signalized Intersections
}

\author{
Mr. Stefan Pidzamecky, Miovision
}

Signalized intersections are at the heart of most urban environments, where pedestrians, cyclists, vehicles, transit, and transport interact. With advancements in video detection capabilities, Miovision is undertaking research and development of multimodal detection and advanced scene understanding. This presentation discussed Miovision's AI data-processing approach for video from Miovision's 360-degree video detection system, SmartSense. The approach supports applications such as multimodal detection and analytics and provides the basis to understand roadside scenarios of people and vehicles. Whereas Miovision is already deployed as a sensor replacement, the sophistication of the video-based technology continues to evolve, approaching the ability to detect, label, and track all objects in the field of view.

\section{Leveraging Sensor-Based Vehicle Position and Speed Information in Traffic Signal Control with Existing Infrastructure Dr. Christopher Day, Iowa State University}

In recent years, many advanced control concepts for traffic signals have been proposed that use enhanced information about vehicle speeds and positions. While it is anticipated that connected vehicles will yield such information, it will require significant time for the technology to proliferate in the fleet to become widely available, and even then, $100 \%$ penetration is not guaranteed due to the (albeit small) proportion of legacy vehicles that will persist. Some detection systems can obtain vehicle position and speed, while some modern controllers are able to communicate information to each other through peer connections. The information developed by advanced detection systems, such as radar, LiDAR, and video cameras, is similar to connected vehicles for control equipment. The conventional and advanced detection systems for intersection sensing are conceptually demonstrated in Figure 7, blocking out portions of the overhead view corresponding to the information gained from the sensing approach. 

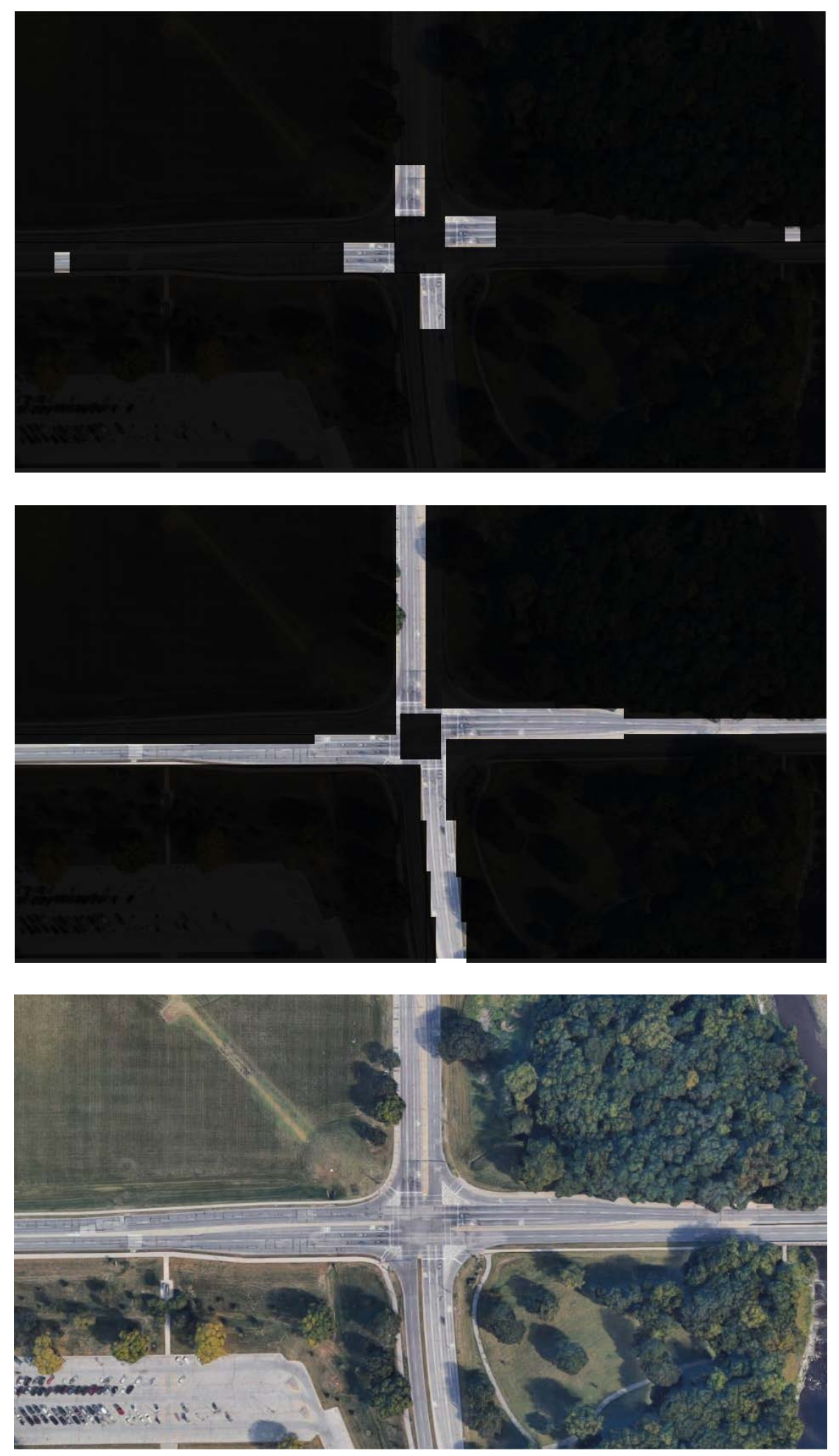

Figure 7. Demonstrations of intersection sensing for conventional and enhanced detection systems; (top) Intersection sensing with conventional detection zones; (middle) Intersection sensing with enhanced detection systems; (bottom) Overarching view of the intersection

In the case study presented, information extracted from existing advanced detection systems is used to intelligently activate side-street signal requests, activating the green phase only when a gap in traffic in the 
primary phase is detected. Although technically simple, this approach advances safety by avoiding dilemma-zone issues related to mainstream vehicles and platoons, and smooths traffic flow by avoiding unnecessary interruptions to platoons on the main arterial. This presentation examined preliminary results from a system that integrates vehicle speed and position data from a radar detection system directly into side-street actuated control logic, using an algorithm that seeks to minimize dilemma-zone impacts and, secondarily, reduce mainline stops. Preliminary results from field implementation at three intersections in Colorado Springs yields a 98\% reduction in dilemma-zone vehicles and a 38\% reduction in mainline stops, based on visual observation of traffic data.

\section{Acknowledgments}

The authors would like to thank all workshop presenters who contributed the research content and diagrams. This workshop summary was authored by the National Renewable Energy Laboratory, operated by Alliance for Sustainable Energy, LLC, for the U.S. Department of Energy (DOE) under Contract No. DE-AC3608GO28308. Funding provided by the U.S. Department of Energy Vehicle Technologies Office (VTO) under the Systems and Modeling for Accelerated Research in Transportation (SMART) Mobility Laboratory Consortium, an initiative of the Energy Efficient Mobility Systems (EEMS) Program. The authors acknowledge Dr. Stan Young of NREL for leading the Urban Science Pillar of the SMART Mobility Laboratory Consortium. The following DOE Office of Energy Efficiency and Renewable Energy (EERE) managers played important roles in establishing the project concept, advancing implementation, and providing ongoing guidance: Prasad Gupte, Erin Boyd, Heather Croteau, and David Anderson. The views expressed in the article do not necessarily represent the views of the DOE or the U.S. Government. The U.S. Government retains and the publisher, by accepting the article for publication, acknowledges that the U.S. Government retains a nonexclusive, paid-up, irrevocable, worldwide license to publish or reproduce the published form of this work or allow others to do so, for U.S. Government purposes. 